\title{
Using full wave seismic modeling to test 4D repeatability for Libra pre-salt field.
}

\author{
Christian Deplante ${ }^{2}$, M. Costa ${ }^{1}$, M. Sebastiao dos Santos ${ }^{1}$, R. de Melo Dias ${ }^{1}$, V. L. Mello ${ }^{1}$, B. Radefeld Meirelles ${ }^{1}$, S. da Silva
} Araujo ${ }^{1}$, D. Chagas Garcia ${ }^{1}$, R.Sansonowski ${ }^{1}$,

${ }^{1}$ Petrobras, ${ }^{2}$ Libra Joint Project Team (Total)

\section{Copyright 2019, SBGf - Sociedade Brasileira de Geofísica}

This paper was prepared for presentation during the $16^{\text {th }}$ International Congress of the Brazilian Geophysical Society held in Rio de Janeiro, Brazil, 19-22 August 2019.

Contents of this paper were reviewed by the Technical Committee of the $16^{\text {th }}$ International Congress of the Brazilian Geophysical Society and do not necessarily represent any position of the SBGf, its officers or members. Electronic reproduction or storage of any part of this paper for commercial purposes without the written consent of the Brazilian Geophysical Society is prohibited.

\begin{abstract}
In the scope of the development of the MERO (Libra NW) project, Libra Joint Project Team and Petrobras are preparing a project of $4 \mathrm{D}$ acquisition on MERO based on a permanent reservoir monitoring (PRM) network to be installed at the seabed in early production phase (less than 2 years after first oil). With more than $65004 \mathrm{C}$ stations planned, such a pre-salt PRM is a novel and ambitious project, the first of its kind in a pre-salt setting. Pre-salt 4D signal is weak and $4 \mathrm{D}$ repeatability noise in particular can be affected by complex salt propagation effects.

Considering these challenges, the costs and reservoir stakes of the project, an extensive full wave acoustic and elastic 4D seismic modeling program has been designed to study geometric repeatability issues as well as prepare future estimation of gas-sensitive 4D attributes and integrate 4D workflow. Preliminary results are discussed
\end{abstract}

\section{Introduction}

Libra pre-salt field, located in Santos basin, contains large resources to be produced over more than two decades, over a relatively compact area a priori favorable to a PRM setup. Pre-salt reservoirs contains distinct carbonate rocks - stromatolites, grainstones and coquinas, all from Aptian age. Reservoirs are thick yet interspersed with igneous rock. Overall, the reservoir units exhibit four orders of magnitude of permeability variations. A key challenge is the very high GOR in Libra NW, with a CO2-rich gas that poses a great challenge for exporting the $\mathrm{HC}$ gas due to restricted economic conditions.

A first Extended Well Test already proved excellent pressure communication but its matching reinforced the role of potentially extended multi-darcyan layers, a potential pathways for early gas breakthroughs and a challenge for gas management. Gas capacity will therefore be the main bottleneck to production and efficient gas management key to project NPV.

The motivations for investing in a frequenct high density/high repeatability 4D scheme on Libra, stems from the anticipated added value of 4D information to directly assist a production drive, based on WAG - alternating water and gas reinjection perhaps combined with other mechanisms. An important aspect of Libra reservoir management is the flexibility of drive obtained using Intelligent Completions Valves (ICVs) with two to three independent levels on injectors and producers. On the 4D side, this implies that on top of the conventional added value of $4 \mathrm{D}$ associated to second wave or infill drilling, the vision of fluid fronts via high quality 4D seismic would also be a key enabler to a more efficient reservoir management via better reservoir understanding and more proactive use of ICVs, with the main objective of preventing or delaying early gas breakthroughs. But one needs to see gas fronts.

$4 D$ signal is not bound by $3 D$ resolution that is solely dependent on useful signal bandwidth: it is equally modulated by $4 \mathrm{D}$ noise. It can detect very subtle dynamic features, on condition of being above $4 \mathrm{D}$ noise, that is dominated by repeatability noise (2): Improving repeatability is the way to go. Yet combining weak signal in thin streaks with the complexity of seismic imaging of pre-salt reservoirs builds a real challenge for 4D detection, a challenge reinforced by the likely necessity to extract 4D AVO attributes such as Ip - Is (Acoustic - Shear impedance) or $\mathrm{dPr} / \operatorname{Pr}$ (Poisson Ratio) to maximize fluid sensitivity and visualize, as shown below, thin gas streaks despite low fluid contrast.

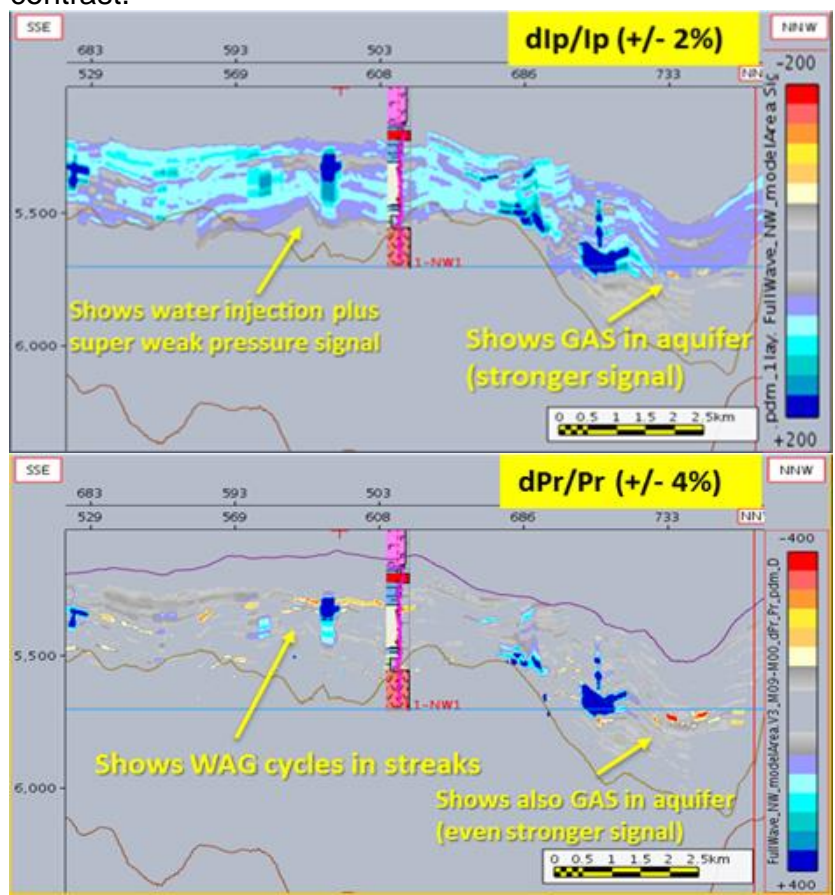

Figure 1 - dlp/lp 4D random line across WAG wells and same with $d P r / \operatorname{Pr} 4 D$ Poisson ratio attribute, from PEM. 
High repeatability such as demonstrated by PRM experience of various operators in the post salt being necessary, a PRM is the anticipated answer to Libra 4D challenge on reception side. But will it be sufficient ? And beyond that, what type of workflow should we use to extract the most usable 4D AVO signal idealized in Fig. 1, with Libra's complex top salt topography having a profound impact on wave propagation and imaging?

To anticipate answers to these difficult questions and provide credible elements of appreciation to Libra asset before making a final investment, we have launched an innovative 4D full wave acoustic and elastic modeling study, incorporating as much realism as practical as of today. The modeling phase of the study has only been running for a few weeks at the time of writing and this expanded abstract will primarily focus on the methodology of model building and modeling, on the setup for testing of various differential acquisition geometries and on calibration and comparisons to real data. However, we will integrate more elements of full wave 4D modeling results as per their availability.

\section{Model building}

Typical 4D feasibilities combine reservoir simulation based on production scenarios with petroelastic modeling to find relevant $4 \mathrm{D}$ observations to address production challenges, as illustrated in Figure 1 for Libra. Following this line a second level study (1) was based on the incorporation of 4D noise according to a noise model and methodology developed by Total (2).It showed that although weak, usable 4D signal could be obtained on Mero, assuming a NRMS level around $2.5 \%$, a pre-salt, a lowfrequency equivalent value, as proposed by PGS(3) to the 3-4\% that has already been obtained by Petrobras with pilot PRM setup in Jubarte post-salt field (4). However, moving from post-salt to pre-salt, seismic propagation becomes as complex as the salt topography implies. In such a setting the 1D convolutional approach appears as a very crude approximation.

Firstly, 1D convolution does not enable to easily integrate important acquisition limitations such as actual source and received density and geometry. Those impact 3D illumination and potentially affect 4D sensitivity to acquisition geometry misfits. Such effects can be modeled accurately by simulating base and monitor acquisition with 3D full wave acoustic or elastic modeling.

Secondly, the 1D convolutional approach does not apprehend the impact of imaging on local blurring, mislocation and lack of amplitude fidelity of the 3D or 4D response, but by actually simulating seismic shots and performing reverse time migration (RTM) with a suitable velocity field, one reproduces these aspects in the simulation results.

To validate the design, it was therefore decided to follow a more ambitious approach, similarly to a paper published in 2016 by Total on a subsalt target (5). Therefore an area of $24.5 \times 24 \mathrm{~km}$, providing good imaging conditions for the widest part of Libra NW (Mero Field) core area, was defined for building a model for full wave seismic modeling. The vertical extension of the model covers from sea surface to a depth of $7 \mathrm{~km}$, deemed sufficient given the quality of the boundary conditions that can be applied to full wave acoustic and elastic propagation.
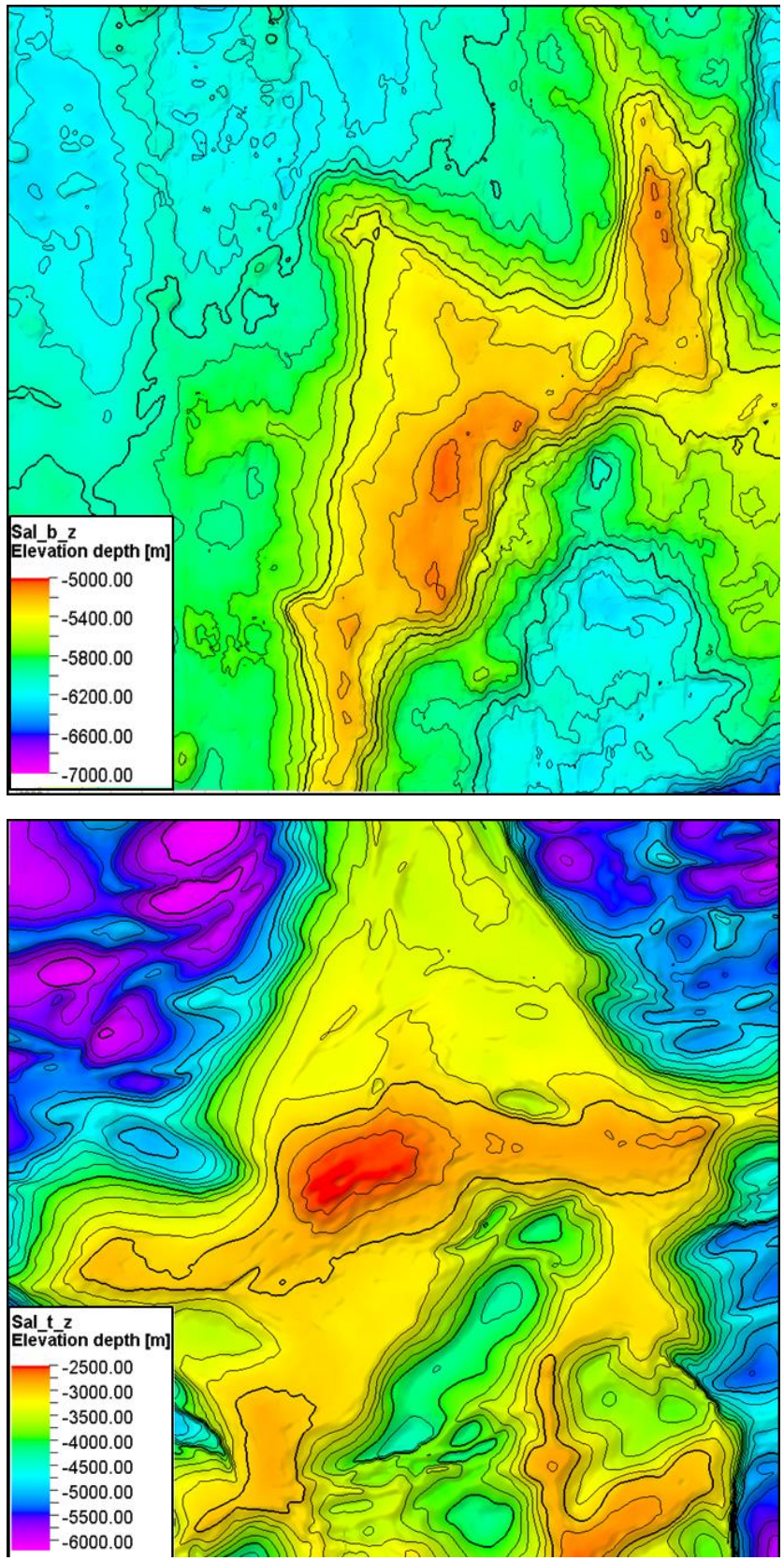

Figure 2 - Model area showing (left) base salt and (right) top salt iso-depth surfaces.

The workflow for model construction has been designed using available well data and the following volumes from Libra's 2016 reprocessing of the streamer SPEC survey:

- FWI velocity volume and tomography volumes of Epsilon and Delta.

- $35 \mathrm{~Hz}$ RTM stack (used from top salt downwards)

- 3 m Kirchoff PSDM (used above top salt)

- Intercept and Gradient extracted form the Kirchoff (used above top salt). 
The modeling workflow is illustrated below.

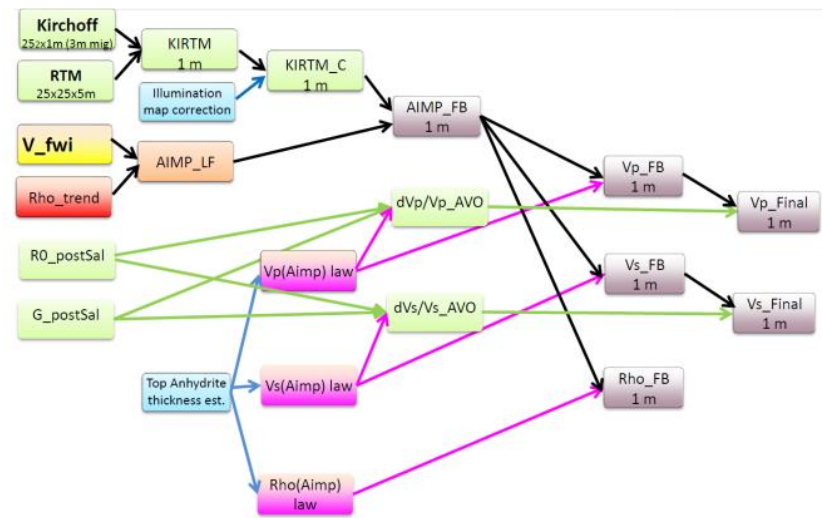

Figure 3-Schematic workflow for elastic model building An hybrid "KIRTM" dataset has first been generated by merging the two stack volumes delimited by top salt as a key surface. It was then corrected for illumination using a first-order volumetric correction consisting of a linear interpolation from constant seabed illumination to variable top salt, then to base salt illumination. Top salt illumination is important to obtain more faithful properties from amplitude. At base salt it was mainly implemented as a first order correction (map-based and not volumetric) to avoid introducing NAZ biases between the petroelastic description of the reservoir properties and the high frequency part of the seismic-driven elastic properties.

The KIRTM stack data has been used to derive the high-frequency part of an impedance model using the Iterdec (C pseudo-impedance generation.

This high frequency part has then been used as a modulation to a low frequency model based on FWI Vp velocities and, for each major lithology interval, Gardnerlike relations relating $R$ ho to $V p$ and $V s$ to $V p$ defined using a set of well logs. The image below compares a Vp section between low frequency, impedance-modulated and AVOmodulated Vp.
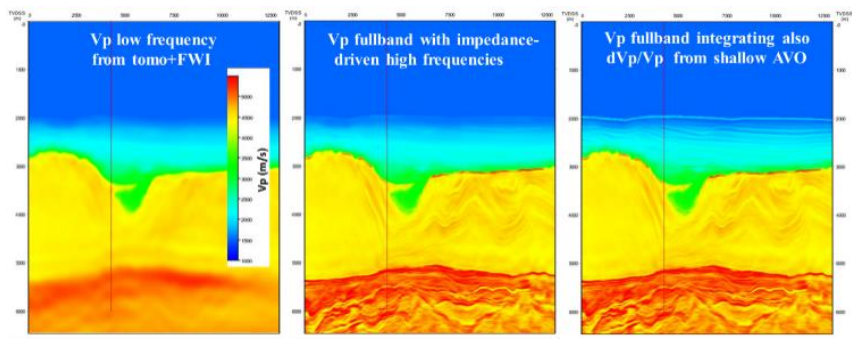

Figure 4: Evolution of Vp volume from FWI, integrating impedance and AVO contributions

Using this approach, a full band acoustic impedance model was first built, then it was mapped from impedance to $\mathrm{Vp}, \mathrm{Vs}$ and density full band cubes using direct mapping from $\mathrm{Al}$ to $\mathrm{Vp}$ and $\mathrm{Vs}$ via chained formulas. Finally, the same interval trend relations were used to derive corrections to $\mathrm{Vp}$ and $\mathrm{Vs}$ to represent shallow AVO anomalies using $R 0$ and Gradient information. The $d V p / V p$ and $\mathrm{dVs} / \mathrm{Vs}$ computed from $\mathrm{R} 0$ and $\mathrm{G}$ were calibrated to the unique pilot hole with shallow $\mathrm{Vp}$ and $\mathrm{Vs}$ sonic logs available on the model area. This is important also to ensure that:

- The wavefronts of reflected PP signal has a realistic complexity due to $\mathrm{Vp}$ anomalies;
The top salt PS reflection wavefront has a realistic complexity due to Vs anomaly.

Especially for a 4D study, the model construction needs to set a major focus on honoring top salt geometry and properties, as this interface is the most critical one influencing:

- P-wave imaging complexity, due to its strong refraction effect and the abrupt topography of top salt in Libra, with steep flanks and several overhangs mainly in the southern half of the study area.

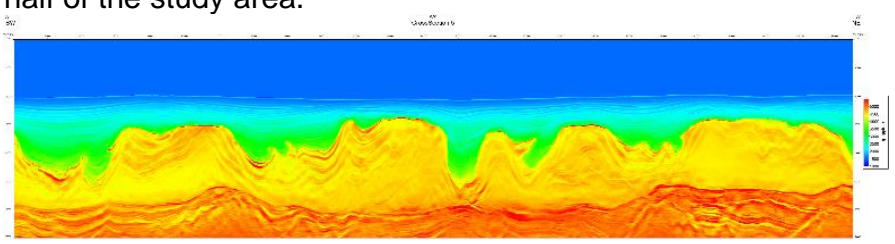

Figure 5: Final Vp model - Random line across overhangs in Southern part of model area

- Elastic propagation complexity, a previous Norsar modeling study has shown the top salt P-S conversion to be a potential strong source of 4D noise interfering with the primary 4D signal, as illustrated by this random line of $\mathrm{P}$ wave velocity model across several overhangs straddling the South of the model area.

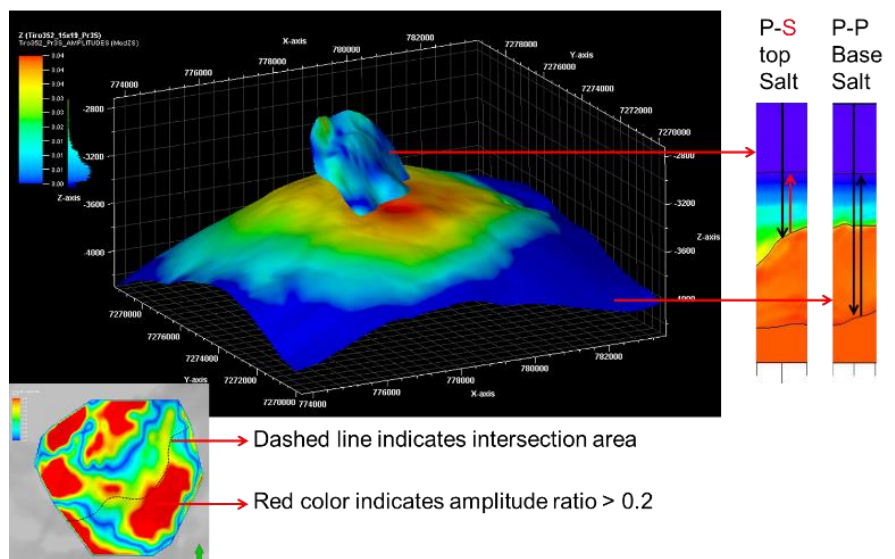

Figure 6: Hodochrons of common seabed gather showing top salt P-S conversion crossing TWT of Base salt PP.

It is therefore crucial that the geometry of top salt and the Vs velocity structure must therefore be adequately represented in the model.

\section{Seismic full wave modeling tools}

The full wave modeling algorithms and computer are those of Advance Geophysical Technology (AGT), using a specific patent on memory allocation and data transfers on GPU architecture (6). Both acoustic and elastic schemes used Convolutional Perfect Matching Layer boundary conditions (CPML), enabling to go down to $1,5 \mathrm{~Hz}$.

\section{Acoustic modeling}

A high-order finite difference acoustic propagation scheme is used. Frequency range is $1.5-43 \mathrm{~Hz}$. At 2.8 points per wavelength with a minimum velocity of $1494 \mathrm{~m} / \mathrm{s}$, a 12.5 $\mathrm{m}$ grid is used for $\mathrm{XY}$ and with a variable depth step.

\section{Elastic modeling}


A high-order spectral element method is used (7). Frequency range is $1.5-25 \mathrm{~Hz}$. The spectral element method enables to use a variable step XYZ grid (hexagonal). The dominant part of the grid is tessellated using a $12.5 \mathrm{~m}$, however the minimum value of Vs with a $12.5 \mathrm{~m}$ grid is 884 $\mathrm{m} / \mathrm{s}$, whereas Vs from extrapolation of shallow well information goes down below $400 \mathrm{~m} / \mathrm{s}$.

The handling of the shallow unconsolidated overburden up to the seabed is a difficulty for shear wave elastic aspects, as Vs becomes very low in very unconsolidated sediments. Fortunately, the code used by AGT enables to create a shallow grid.

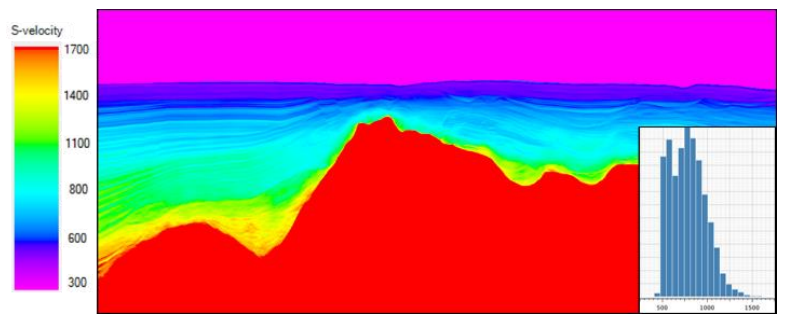

Figure 7: Shallow Vs structure and histogram. S-Velocities (zero in water) 300 to $1700 \mathrm{~m} / \mathrm{s}$.

\section{Various differential acquisition geometry tests}

Both source and receiver geometry settings and their perturbations have been directly taken from the preplots and postplots of the OBN survey conducted over Libra NW from mid-2017 to mid-2018, and for the PRM, used the last available preliminary layout at the time of launching the study.

The project setup has been designed to enable the following comparisons:

- PRM versus PRM (where differences come only from shot side. Shot statistics of postplots minus preplots of OBN acquisition were used as per Figure 8.

- PRM versus OBN assuming inline interpolation of signal to have only a crossline residual misfit

- OBN versus OBN as a comparison standpoint to the PRMPRM

All these comparisons will be available with either with or without the same source location misfits used for the PRMPRM geometry misfit assessment.

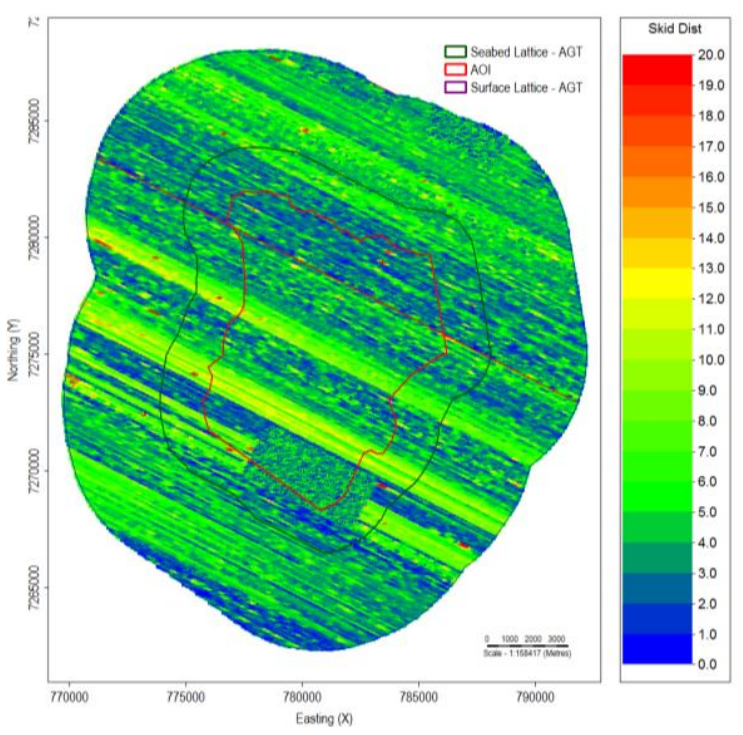

Figure 8: Statistics of errors for shot repeat misfits.

\section{Calibration of synthetic versus 4D Noise test.}

A 4D noise test has also been carried out during the OBN survey in a denser area and will be simulated. Its aim was to provide calibration data for the PRM - OBN case as well as for the OBN-OBN case. The dispersion of relative positions of collocated nodes (R2) with respect to the main nodes (R1) follows two predefined statistics as per figure below, showing a distribution elongated to $+/-25 \mathrm{~m}$ on either side to represent the 4D noise associated with a $50 \mathrm{~m}$ PRM station sampling randomly a $500 \mathrm{~m}$ OBN station reference with a smaller crossline error $(+/-10 \mathrm{~m})$, and a more circular random error for OBN-OBN relocation. The test area was adequately located in the shot overlap area of southern and northern swath overlap of the OBN survey so that for each node, the effect of difference between two quasi-identical source locations $\mathbf{S} 1$ and $\mathbf{S} 2$ can be tested.

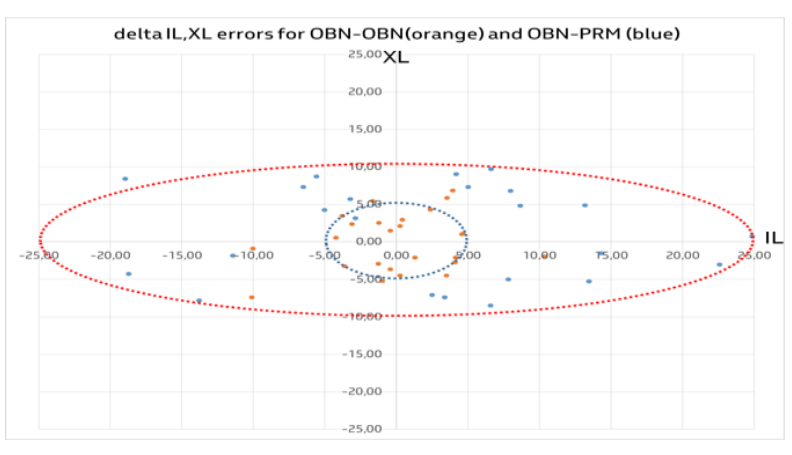

Figure 9: Post-plots of relocation errors for two populations of nodes.

Three differential pairs (S2R1-S1R1, S1R2-S1R1 and S2R2-S1R1) extracted from this noise test will enable to calibrate the relative impact of the geometry misfit with respect to the other sources of non-repeatability, or rather their residual part not absorbed by 4D preprocessing and regularization:

- fluctuations of source signature 
- tide and water velocity residual effects

- residual clock drift and sensor orientations plus coupling variations (for OBN acquisitions)

Such effects will be present in the real data but not in the modeling. They were not modeled, in the absence of credible models to describe such fluctuations.

\section{Comparisons to real data}

The locations of 8 nodes from the real OBN survey have been provided. Preliminary gather comparisons show a satisfactory mismatch to real data even before deghosting on the synthetic side.
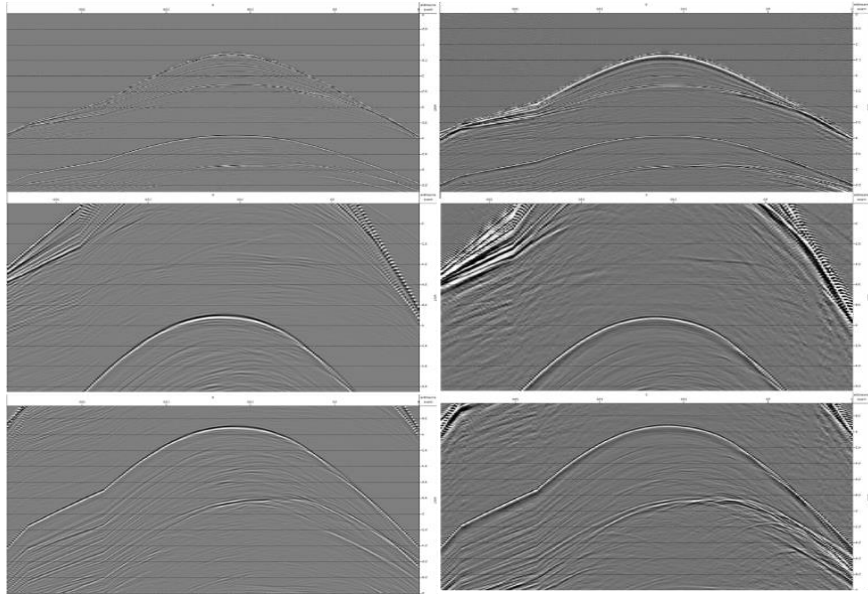

Figure 10: Preliminary comparisons of real (right) vs acoustic modeling (left) of OBN gather line

\section{Preliminary 4D signal}

Whereas the acoustic data are modeled up to a maximum frequency of $43 \mathrm{~Hz}$, they contain neither the 4D AVO effects at reservoir level, nor the elastic components of 4D noise. The elastic data contains both, but full wave elastic modeling is so computer intensive that we had to limit maximum frequency to $25 \mathrm{~Hz}$.

A prelimination $Q C$ of the acoustic 4D signal available at the level of an individual seabed station shows that the expected velocity effect of a depletion around 25 bars, creating 70 to $100 \mathrm{~m} / \mathrm{s}$ (around $1.5 \%$ ) velocity change over the thick reservoir interval, produces a timeshift of a small fraction of one ms but can be easily detected in the context of highrepeatability $4 \mathrm{D}$ signal.
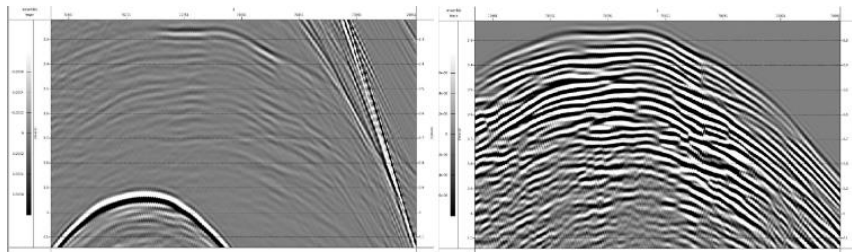

Figure 11: Zoom on reservoir interval for (left) $4 D$ baseline OBN gather, (right) $100 \times 4 D$ difference.

\section{Preliminary findings}

The difficult question that this study tries to address is the issue of the level of repeatability noise to be expected in the particular setting of a permanent seabed reception over a presalt lacustrine reservoir with complex salt geometry. We integrate the results of a 4D noise test to calibrate modeled repeatability noise. The underlying question is the question of weak (or super-weak) signal detectability, direcly linked to 4D noise characteristics, which we are gaining insights into, based on these preliminary results. One of the interesting venues of this work will be the capability of using these synthetic data to prepare and optimize the $4 \mathrm{D}$ processing, estimation of 4D attributes as well as their integration into reservoir management.

\section{Acknowledgments}

The authors wish to thank Petrobras and partners PPSA, Shell, Total, CNOOC and CNPC for their support of the study and permission to show these results, as well as AGT who has shown strong commitment to this study from day 1 onwards. Special thanks are also deserved to Ricardo Augusto Rosa Fernandes and Paulo Roberto Schroeder Johann for their longstanding support for Libra PRM.

\section{References}

(1) Submitted for EAGE 2019 - 4D Petroelastic Modeling for a Brazilian Pre-Salt Field: What to Expect From Interpretation? V.L. Mello1 M.S. Santos, R.M. Penna, J.A. Rosseto, C. Deplante

(2) Hubans, C. [2016]. 4D Noise Understanding to Validate 4D Anomalies. In: 78th EAGE Conference and Exhibition. Viena, Austria, expanded abstracts

(3) Didier Lecerf - Repeatability measure for broadband 4D seismic - Didier Lecerf, Jon Burren, Ed Hosges and Caio Barros, PGS - SEG 2015, expanded abstracts

(4) Permanent Reservoir Monitoring - 4D Quantitative Interpretation - Yermek Balabekov (PGS), Marcos Sebastiao (Petrobras), Ruan Couto (Petrobras), Paula Dariva (Petrobras), Wilson Lisboa (Petrobras), Cyrille Reiser (PGS), Paulo Johann (Petrobras) SBGf 2017, Expanded Abstract.

(5) Advanced subsalt imaging results and 4D seismic design evaluation, SEG 2016 Expanded Abstracts, C. Geream H. Baudon, J. Colonge, R. Lencrerot, $P$. Solans.

(6) US Patent 8,972,234 B2 (Mar. 3, 2015, AGT) Method and system to reduce: memory requirements, device-to-host transfer bandwidth requirements, and setup time, for seismic modeling on graphics processing units

(7) Large scale seismic modeling for SEAM Phase II using the finite element method on GPUs - Lijian Tan*, Yu Zhang, Wenyi Hu, Advanced Geophysical Technology, Inc., Michael Oristaglio, Yale and SEAM Phase /I Project Manager, SEG SEAM Abstract, 2017 